\title{
Capstone Design en los Programas de Ingeniería: Una Revisión Sistemática
}

\author{
Capstone Design in Engineering Programs: A Systematic Review \\ Camilo Andrés Urrea Merchán ${ }^{1}$ \\ https://orcid.org/0000-0003-4416-9951
}

Johann Alberto Duque Mogollón ${ }^{2}$

https://orcid.org/0000-0001-6341-118X

Edelberto Vásquez González ${ }^{3}$

https://orcid.org/0000-0001-9758-3211

Universidad Industrial de Santander, Colombia

Recibido:10-06-2021

Aceptado: 29-08-2021

\section{Cita Recomendada}

Urrea, C., Duque, J. y Vásquez, E., (2021). Capstone design en los programas de ingeniería: Una revisión sistemática. Hamut' ay, 8 (2), 31-42, http://dx.doi.org/10.21503/hamu.v8i2.2288

\begin{abstract}
Resumen
Los programas de ingeniería reconocen cada vez más la importancia de incluir en sus currículos, cursos de experiencias de diseño (Capstone Design, por sus términos en inglés) como estrategia formadora y enriquecedora para los futuros profesionales. El presente estudio identificó la evolución de este tipo de cursos en el período comprendido entre 1989 y 2020. Para ello, se realizó una revisión de 33 artículos publicados en SCOPUS. Se analizaron seis lustros con la ayuda de la herramienta VOSviewer para la identificación de temas concurrentes y emergentes. Los resultados mostraron que, los cursos "Capstone Design", independientemente de la disciplina en la cual se apliquen, tienen como objetivo brindar herramientas para que los estudiantes afronten un reto profesional aplicado. En el primer lustro analizado el tema más concurrente fue estudiante y la propuesta de diversas estrategias para fortalecer el aprendizaje; en el segundo periodo, el interés estuvo en apoyarse en la experiencia de la industria para satisfacer muchas de sus necesidades; el tercer periodo se caracterizó por resaltar la importancia del mentor o profesor como factor de éxito en los cursos; el cuarto periodo, recogió las experiencias vividas en cursos anteriores para plantear mejoras; el quinto periodo, proveyó un marco conceptual y referenció habilidades requeridas por la industria; para finalizar, el sexto periodo se centró en los problemas que los estudiantes tienen al desarrollar proyectos en los cursos. Se espera que los resultados de esta revisión, aporte insumos a los programas colombianos de ingeniería, para la reorganización de cursos relacionados con experiencias de diseño.
\end{abstract}

Palabras Clave: Capstone Design, Syllabus, VOSviewer, Experiencia en Diseño, Experiencia Maestra.

1. Semillero de investigación Modelado y Gestión de la Construcción. Bachiller técnico. camilo2164189@correo.uis.edu.co, santoprimos@gmail.com.

2. Semillero de investigación Modelado y Gestión de la Construcción. Bachiller técnico. johann2170247@correo.uis.edu.co, johann1919@hotmail.com.

3. Coordinador BIM. Semillero de investigación Modelado y Gestión de la Construcción. Ingeniero Civil; Máster en BIM. edelberto. vasquez@correo.uis.edu.co, betovasquezz3@hotmail.com. 


\begin{abstract}
Engineering programs increasingly recognize the importance of including design experience courses (Capstone Design) in their curricula as an educational and enriching strategy for future professionals. The present study identified the evolution of this type of course in the period between 1989 and 2020. For this, a review of 33 articles published in SCOPUS was carried out. Six lustrums were analyzed with the help of the VOSviewer tool to identify concurrent and emerging issues. The results showed that the "Capstone Design" courses, regardless of the discipline in which they are applied, aim to provide tools for students to face an applied professional challenge. In the first five years analyzed, the most concurrent theme was student and the proposal of various strategies to strengthen learning; In the second period, the interest was in relying on the experience of the industry to satisfy many of its needs; the third period was characterized by highlighting the importance of the mentor or teacher as a success factor in the courses; the fourth period, collected the experiences lived in previous courses to propose improvements; the fifth period, provided a conceptual framework and referenced skills required by the industry; Finally, the sixth period focused on the problems that students have when developing projects in the courses. The results of this review are expected to provide inputs to Colombian engineering programs for the reorganization of courses related to design experiences.
\end{abstract}

Key words: Capstone Design, Syllabus, VOSviewer, Design Experience, Master Experience.

\section{Introducción}

El Consejo de Acreditación para Ingeniería y Tecnología (ABET, por sus siglas en inglés) define el diseño de ingeniería, como el proceso de concebir un sistema, componente o proceso para satisfacer una serie de necesidades (ABET 2020). En concordancia, los programas de ingeniería deben asegurar a los estudiantes una experiencia maestra de diseño, que posibiliten la integración de principios, conceptos y técnicas vistas en cursos previos, como parte de la estructura de sus currículos (Marin, Armstrong, y Kays 1999). Actualmente, los programas de ingeniería revisan continuamente sus currículos y sus estrategias de formación, para el cumplimiento social de su misión; estrategia que ha de responder a la necesidad de preparar en adecuadamente a los futuros profesionales en prácticas de la ingeniería enfocadas hacia la solución de problemas reales, la aplicación de conceptos fundamentales de ingeniería y el desarrollo de habilidades. Así, los cursos enfocados hacia experiencias de diseño, surgen como alternativas para brindar a los estudiantes una formación enriquecedora.

Un curso Capstone Design, generalmente se apoya en el aprendizaje basado en proyectos, lo que demanda un gran compromiso de acompañamiento por parte del cuerpo docente del programa, para ofrecer posibilidades significativas de aprendizaje y colaboración (Todd, Sorensen y Magleby 1993). De esta manera, los estudiantes de ingeniería reciben una enseñanza fundamentada en el diseño, el trabajo en equipo, la experiencia práctica y la aplicación de conocimientos adquiridos en cursos previos de su malla curricular (Bass y Begovic, 1997; Milke, Paul y Loorey, 2015). En muchos casos, estos cursos se caracterizan por la participación de empresas o agentes externos a la universidad, con la finalidad de que los futuros egresados experimenten con proyectos de la vida real, y así adquieran habilidades que les permitan manejarlos, ser autodidacta, gestionar equipos de trabajo, gestionar proyectos y comunicarse (Joseph, 1999; Mertz, 1997).

Por las razones hasta aquí descritas, como aporte a la formación en ingeniería, este estudio tuvo como objetivo realizar una revisión bibliográfica sobre los cursos de "Capstone Design", para identificar sus características, propósitos y mejoras, para así determinar la evolución de las propuestas curriculares a través del tiempo.

\section{Cursos “Capstone Design”}

Los cursos de "Capstone Design" están orientados a dar herramientas y a desarrollar las capacidades necesarias para que los estudiantes lleven a cabo prácticas de ingeniería real (Milke, 
Paul y Koorey, 2015), como parte de los planes de estudio de pregrado. En ellos es posible sintetizar las temáticas vistas en cursos anteriores y ponerlas en práctica a través de la aplicación de técnicas y habilidades profesionales en proyectos (Paretti et al., 2020); y de ponerse en contacto con entes externos, que exigen el esfuerzo de los estudiantes durante el desarrollo de los proyectos, lo que incluye la planificación y control de los mismos (Banios, 1992).

Con los cursos de "Capstone Design", se busca subsanar las falencias que tienen los nuevos profesionales para adaptarse al ámbito laboral, ya que, la aplicación de la teoría a la práctica no es sencilla ni fácil de orientar por la cantidad de variables que implican el relacionar la una con la otra; por esto, se vuelven una pieza muy importante en los procesos de acreditación de programas de ingeniería, dado el interés de los empleadores se interesan en contratar egresados de programas con dichas acreditaciones.

\section{Método}

La revisión bibliográfica realizada el 11 de febrero de 2021, se ciñó a las recomendaciones dadas para una revisión sistemática tipo Scoping Review (The Joanna Briggs Institute, 2015). Se realizó una búsqueda bibliográfica en la base de datos SCOPUS, con la ecuación de búsqueda: TITLEABS-KEY (("CAPSTONE DESIGN" OR "MAJOR DESIGN EXPERIENCE") AND "ENGINEERING EDUCATION") AND (LIMIT-TO (DOCTYPE, "ar")). Con base en esta ecuación se obtuvo una muestra inicial de 116 artículos, los cuales fueron clasificados en cinco grandes temáticas: Evaluación, Proyecto, Currículo, Habilidades, Programa del curso, y Otros (ver Tabla 1).

Tabla 1. Temáticas iniciales de los documentos $(\mathrm{N}=116)$

\begin{tabular}{lcc}
\hline \multicolumn{1}{c}{ TEMA } & No Documentos & Porcentaje \\
\hline Evaluación & 15 & $13 \%$ \\
Proyecto & 24 & $21 \%$ \\
Currículo & 18 & $16 \%$ \\
Otros & 10 & $9 \%$ \\
Habilidades & 10 & $9 \%$ \\
Programa del curso & 39 & $34 \%$ \\
\hline Total & 116 & $100 \%$ \\
\hline
\end{tabular}

Nota: Fecha de la búsqueda en SCOPUS febrero 11 de 2021. Fuente: Elaboración propia (2021)
De los 116 inicialmente identificados, se seleccionaron aquellos artículos clasificados dentro de la temática Programa del curso, para describir su evolución a lo largo del tiempo. Así se llegó a 39 artículos que hablaban sobre la estructura del programa del curso, seis de los cuales no se pudieron descargar, motivo por el cual, la muestra final del estudio quedó conformada por 33 artículos.

\section{Procedimiento}

El análisis final de la revisión de 33 artículos que hacen referencia a las características de los programas del curso "Capstone Design", indica que el 94\% (31/33) de estos documentos se encontraron en revistas especializadas, con clasificación SCImago, y el restante 6\% $(2 / 33)$ fueron actas de congreso que no tenían clasificación en este sistema. De acuerdo con la clasificación SCImago, el 74\% (23/31) de las revistas analizadas en este estudio están en cuartil Q1, y un 23\% (7/31), en cuartil Q2. Adicionalmente, se encontró que el 55\% (17/33) corresponden a estudios publicados en revistas en ingeniería, especializadas en educación (RIEE), $y$ el 39\% (12/33), en revistas especializadas en educación en ingeniería [REI] (ver Tabla 2).

Tabla 2. Tipo de revista donde se publicaron los estudios $(\mathrm{N}=33)$

\begin{tabular}{|c|c|c|c|}
\hline \multicolumn{2}{|c|}{ Tipo de Revista } & \multicolumn{2}{|c|}{ No Documentos Porcentaje } \\
\hline CONG & Actas de Congresos & 2 & $6 \%$ \\
\hline $\mathrm{RE}$ & Revista especializada en educación & 1 & $3 \%$ \\
\hline REI & $\begin{array}{l}\text { Revista especializada en educación } \\
\text { en ingeniería }\end{array}$ & 12 & $36 \%$ \\
\hline RI & Revista especializada en ingeniería & 1 & $3 \%$ \\
\hline RIEE & $\begin{array}{l}\text { Revista en ingeniería especializada } \\
\text { en educación }\end{array}$ & 17 & $52 \%$ \\
\hline Total & & 33 & 100 \\
\hline
\end{tabular}

Fuente: Elaboración propia (2021)

La muestra final compuesta por artículos publicados entre 1989 y 2020, se agrupó en periodos de 5 años (ver Tabla 3). 
Tabla 3. Clasificación de búsqueda bibliográfica por periodos.

\begin{tabular}{lcc}
\hline PERIODOS & No Documentos & Porcentaje \\
\hline Primer periodo [1989-1995] & 7 & $21 \%$ \\
Segundo periodo [1996-2000] & 8 & $24 \%$ \\
Tercer periodo [2001-2005] & 6 & $18 \%$ \\
Cuarto periodo [2006-2010] & 4 & $12 \%$ \\
Quinto periodo [2011-2015] & 2 & $6 \%$ \\
Sexto periodo [2016-2020] & 6 & $19 \%$ \\
\hline Total & 33 & $100 \%$ \\
\hline
\end{tabular}

Fuente: Elaboración propia (2021)

Una vez clasificados los artículos en los seis periodos, se analizó cada uno de ellos, en búsqueda de sus características y los cambios más relevantes en sus propósitos durante los periodos de tiempo observados. Para esto, se usó la herramienta VOSviewer, que permitió hacer una revisión más eficiente de los artículos en cada periodo. Con esta herramienta se identificaron las características a partir de los temas que más aparecían en cada periodo y las relaciones entre ellos, lo que permitió obtener un panorama general desde las palabras claves más concurrentes (Figura 1).

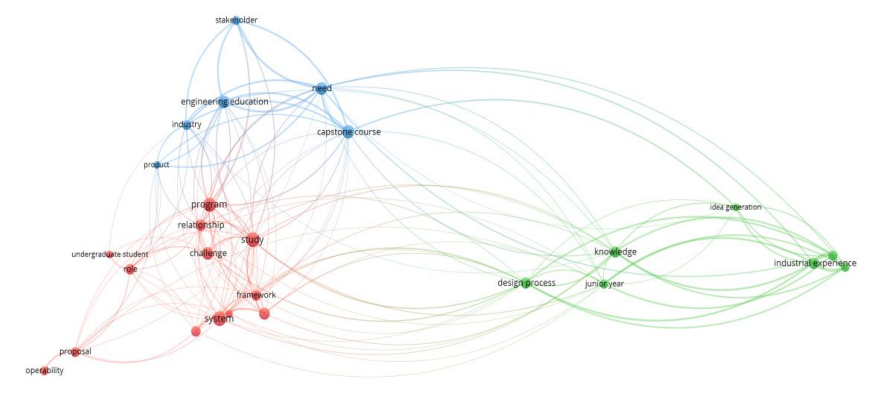

Figura 1. Análisis VOSviewer.

Fuente: Elaboración propia (2021)

Además de encontrar características emergentes, con el estudio se pudieron establecer relaciones entre los primeros cursos y los actuales, proceso que posibilitó la descripción de la evolución de los propósitos y mejoras de los cursos de "Capstone Design" y su estructuración en los programas de ingeniería.

\section{Resultados}

A continuación, se describirán las características más importantes encontradas en cada período. En el primer periodo comprendido entre 1989 y 1995, en la revisión bibliográfica se analizaron un total de 7 artículos, descritos en Tabla 4.

Tabla 4. Artículos periodo 1989-1995

\begin{tabular}{ll}
\hline Título & \multicolumn{1}{c}{ Cita } \\
\hline $\begin{array}{l}\text { Designing the design engineer } \\
\text { University-Industry Technology Interchange } \\
\text { Through a Unique Engineering Projects Course }\end{array}$ & (Liebman, 1989) \\
Civil engineering capstone design course & (Andersen, 1992) \\
$\begin{array}{l}\text { An Engineering Practices Course } \\
\text { Designing a Senior Capstone Course to Satisfy }\end{array}$ & (Banios, 1992) \\
Industrial Customers & (Todd, Sorensen, y Magleby, \\
Integrated capstone design experience & 1993) \\
On Effective Methods to Teach Mechanical Design & (Harris y Jacobs, 1995) \\
\hline
\end{tabular}

Fuente: Elaboración propia (2021)

Los estudios de este período fueron realizados en Norteamérica, donde los cursos de "Capstone Design" tuvieron una duración de 1 a 3 semestres, y pertenecían a programas de ingeniería mecánica, ingeniería de sistemas, ingeniería eléctrica e ingeniería civil. El análisis con VOSviewer permitió identificar como palabra más concurrente "estudiante" (Figura 2). En este periodo también se realizó un cuestionamiento a la educación en ingeniería y las debilidades que poseían los ingenieros recién egresados al ingresar a la industria, al mostrar poco conocimiento sobre cómo diseñar y cómo gestionar los proyectos (Liebman, 1989). Otras de las debilidades detectadas, fueron el inadecuado enfoque dado a la estructura de los proyectos, la falta de métodos de diseño que permitieran alcanzar mejor calidad al momento de realizar estos, como también, las falencias comunicativas evidenciadas en las dificultades observadas al momento de hacer presentaciones orales efectivas, estructuradas y concisas (Todd, Sorensen, y Magleby, 1993). Aunque se identificó la necesidad de una ingeniería integrada, no se trabajó en estrategias concretas para asegurar su integración. 


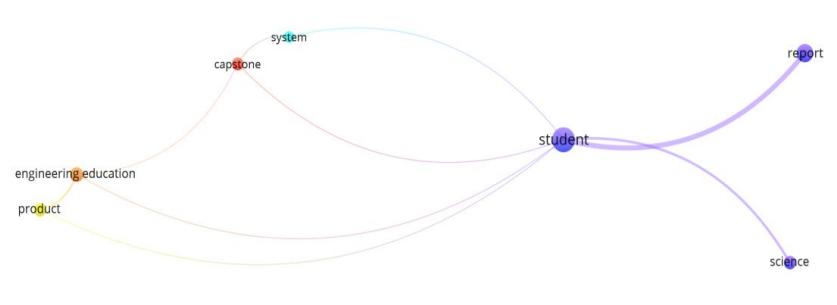

B vosviewer

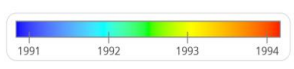

Figura 2. Análisis VOSviewer primer periodo.

Fuente: Elaboración propia (2021)

De otro lado, varios autores resaltan algunos beneficios de los cursos de "Capstone Design", ya que los estudiantes al esforzarse más en el desarrollo de los diseños y al necesitar utilizar gran parte de los conocimientos adquiridos en su plan de estudios al momento de resolver problemas reales de ingeniería, adquieren mayor conocimiento sobre aspectos profesionales de la ingeniería, a través de los estudios, las conferencias y las experiencias obtenidas. Esto les permitió comprender que existen múltiples soluciones a un problema real en ingeniería y, reforzar habilidades como el trabajo en equipo, la interacción entre estudiantes, la comunicación y el probar soluciones tanto teóricas como en campo (Banios, 1992; Harris y Jacobs, 1995).

En el segundo periodo comprendido entre 1996 y 2000, en la revisión bibliográfica se analizaron un total de 8 artículos, como se detalla en Tabla 5 .

Tabla 5. Artículos periodo 1996-2000

\begin{tabular}{|c|c|}
\hline Título & Cita \\
\hline Instrumentation and Communication Modules on & (Mehta y Gronhovd, 1996) \\
\hline CD-ROM's for Enriching Engineering Education & \\
\hline A Capstone Design Course & (Mertz, 1997) \\
\hline $\begin{array}{l}\text { A senior design course bridging power systems and } \\
\text { power electronics: Power quality impacts of electric } \\
\text { vehicle charging }\end{array}$ & (Bass y Begovic, 1997) \\
\hline Elements of an optimal capstone design experience & (Marin, Armstrong, y Kays, 1999) \\
\hline Using Contests to Teach Design to EE Juniors & (Gregson y Little, 1999) \\
\hline $\begin{array}{l}\text { An undergraduate computer engineering rapid } \\
\text { systems prototyping design laboratory }\end{array}$ & (Hamblen et al., 1999) \\
\hline A capstone computer engineering design course & (Richard, Taylor, y Zar, 1999) \\
\hline Coordinating Diverse Set of Capstone Design & (Joseph, 1999) \\
\hline
\end{tabular}

Fuente: Elaboración propia (2021)
Los artículos mencionados correspondieron a estudios realizados en Norteamérica, donde los cursos de "Capstone Design" tuvieron una duración de 1 a 2 semestres, y correspondieron a programas de ingeniería mecánica, ingeniería de sistemas, ingeniería eléctrica e ingeniería civil. El análisis con VOSviewer permitió identificar como palabras más concurrentes "estudiante", "curso" y "experiencia" (Figura 3).

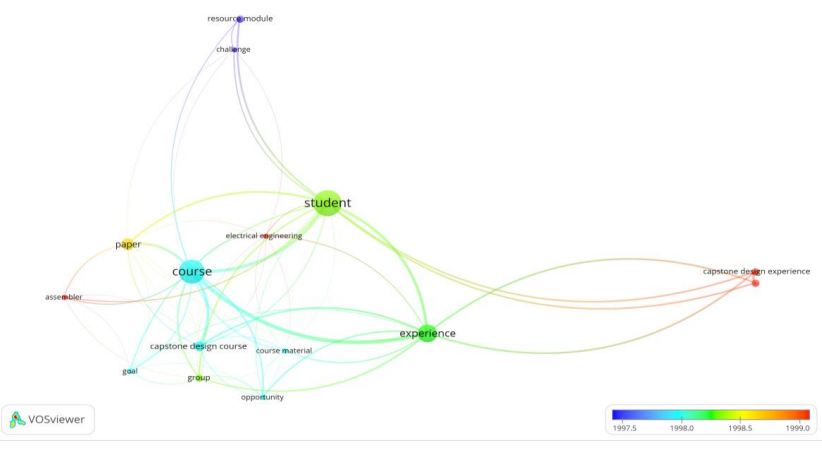

Figura 3. Análisis VOSviewer segundo periodo. Fuente: Elaboración propia (2021)

En este periodo se reconocen algunas características constructivas producidas por el desarrollo dealgunos cursos de "Capstone Design". La afinidad a éticas sociales, económicas, legales y seguridad que no se trataban en el contexto del diseño de ingeniería y la práctica profesional en otros cursos (Mertz, 1997). Las técnicas de motivación empleadas por los instructores con la finalidad de hacer que los estudiantes se apropiaran del proyecto y lo finalizaran con gran éxito. La flexibilidad en las técnicas utilizadas para que los estudiantes tuvieran la oportunidad de fracasar y tener éxito. Los proyectos como transición del estudio a la industria, mediante la experiencia de un diseño en el entorno académico (Mertz, 1997). La adquisición de habilidades técnicas y procedimientos fundamentales para la realización de proyectos de ingeniería mediante aprendizaje autónomo, en el que debe desarrollar habilidades adicionales de comunicación oral y escrita (Joseph, 1999). También se resalta la necesidad de preparación, administración, ejecución, y evaluación de los cursos y de los proyectos; se reconoce la importancia del rol del cliente, motivo por el cual al finalizar el proyecto se pedía llenar una encuesta evaluando a los 
estudiantes. Estos cursos debían contar con tres actores importantes los cuales eran: Instructor, estudiante y cliente (Marin, Armstrong, y Kays, 1999).

Es así que para desarrollar un buen curso de "Capstone Design", se hace necesario cumplir con las siguientes condiciones: ser seguro, brindar la oportunidad de adquirir mayor conocimiento de los hechos y los procedimientos, ejercitar el juicio de ingeniería, fomentar la creatividad, proporcionar éxito acorde con el cuidado en el diseño, permitir muchas estrategias con niveles de éxito, no requerir una infraestructura significativa, ser fácil de entender $y$, poder ser evaluado con una puntuación sencilla. Es así que para poder abordar adecuadamente la ingeniería se requiere conocimiento fáctico, conocimiento de procedimientos, capacidad de identificar conceptos clave, capacidad de adquirir nuevos conocimientos y juicio para utilizar información incompleta/contradictoria (Gregson y Little, 1999).

Otras experiencias positivas, resaltan la percepción positiva y útil de estos cursos por parte de los egresados, al ser fuente de proyectos innovadores y patentes que pueden ser usados por la industria (Hamblen et al., 1999; Joseph, 1999).

En el tercer periodo comprendido entre 2001 y 2005, en la revisión bibliográfica se analizaron un total de 6 artículos, como se detalla en Tabla 6.

Tabla 6. Artículos periodo 2001-2005

\begin{tabular}{ll}
\hline Título & \multicolumn{1}{c}{ Cita } \\
\hline $\begin{array}{l}\text { Cooperative Learning Instructional Activities in a } \\
\text { Capstone Design Course }\end{array}$ & $\begin{array}{l}\text { (Russ Pimmel, 2001) } \\
\text { Pan-Mentoring' as an Effective Element of Capstone }\end{array}$ \\
$\begin{array}{l}\text { Design Courses } \\
\text { University Methodology for Internetworking }\end{array}$ & (Abler, Owen, y Riley, 2003) \\
$\begin{array}{l}\text { Principles and Design Projects } \\
\text { Elements of a successful capstone course } \\
\text { considering the needs of stakeholders }\end{array}$ & (Todd y Magleby, 2005) \\
$\begin{array}{l}\text { Teaching Digital Design to Computing Science } \\
\text { Students in a Single Academic Term }\end{array}$ & (Amaral, Berube, y Mehta, 2005) \\
Bridging the Gap between Academics and Practice: & (Hanna y Sullivan, 2005) \\
A Capstone Design Experience &
\end{tabular}

Fuente: Elaboración propia (2021)

Los estudios de este periodo fueron realizados en Norteamérica, donde los cursos de "Capstone Desing" tuvieron una duración de 1 a 2 semestres, en programas de ingeniería eléctrica, ingeniería mecánica, ingeniería informática, ingeniería e ingeniería civil. El análisis con VOSviewer identificó palabras concurrentes como "proyecto", "industria" y "metodología" (Figura 4).

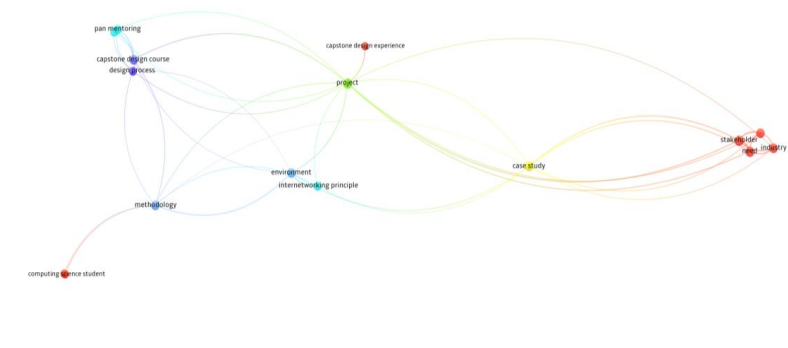

Figura 4. Análisis VOSviewer tercer periodo. Fuente: Elaboración propia (2021)

Los cursos en este periodo buscaban la participación de varios profesores del programa y mejorar la relación profesor-estudiante, para que los estudiantes pudieran completar su experiencia en el área de su interés (Russ Pimmel, 2001). El profesor comienza a verse como un mentor que facilita la comunicación; que asegura el flujo continuo de ideas, dibujos y bocetos, elementos integrales del proceso de diseño; que promueve el trabajo en equipo como elemento esencial en el proceso de enseñanza. Así, el mentor genera una atmosfera que apoya la toma de riesgos, la manera en la cual se evalúan los estudiantes, el aprendizaje independiente, la construcción de conocimiento y la resolución de problemas (Ekwaro-Osire, 2003).

Los aspectos que se mejoraron en cursos de "Capstone Design" fueron: aplicar el conocimiento académico a un problema práctico, la integración de varias disciplinas en un problema de diseño abierto del mundo real, la promoción del trabajo en equipo al permitir que los estudiantes trabajen en grupos con miembros de diferentes competencias y responsabilidades, la mejora de las habilidades de comunicación verbal y escrita, la promoción de la interacción de los estudiantes con profesionales externos y usar la tecnología para resolver problemas (Hanna y Sullivan, 2005).

Otros aspectos positivos encontrados fueron: el estudiante se vuelve más autodidacta (Amaral, Berube, y Mehta, 2005); el estudiante se involucra 
con profesionales en ejercicio; en los diseños se involucran conceptos de factibilidad, ingeniería de valor, preparación de cronogramas de construcción, estimación de costos, y simulación de creación de empresas conformadas por estudiantes con diferentes capacidades y aptitudes complementarias entre si (Hanna y Sullivan 2005). Así mismo, en el desarrollo del curso se identifican las partes interesadas; se determinan sus necesidades, deseos y valores; se plantean propuestas que satisfagan las necesidades de las partes interesadas; se evalúa y selecciona la mejor propuesta; se realiza un diseño general y detallado; se prueba y perfecciona el diseño (Todd y Magleby, 2005).

En el cuarto periodo comprendido entre 2006 y 2010, en la revisión bibliográfica se analizaron un total de 4 artículos, como se detalla en Tabla 7.

Tabla 7. Artículos periodo 2006-2010

\begin{tabular}{ll}
\hline Título & \multicolumn{1}{c}{ Cita } \\
\hline $\begin{array}{l}\text { Effects of industrial experience and coursework during } \\
\text { sophomore and junior years on student learning of } \\
\text { engineering design }\end{array}$ & (Bailey 2007) \\
$\begin{array}{l}\text { Integrating graduate design coaches in undergraduate } \\
\text { design project teams }\end{array}$ & (Powers y Summers 2009) \\
$\begin{array}{l}\text { Directional-based cellular e-commerce: Undergraduate } \\
\text { systems engineering capstone design Project }\end{array}$ & (Al-Rizzo et al. 2010) \\
$\begin{array}{l}\text { Teaching "operability" in undergraduate chemical } \\
\text { engineering design education }\end{array}$ & (Marlin 2010) \\
\hline
\end{tabular}

Fuente: Elaboración propia (2021)

Los artículos correspondieron a estudios en Norteamérica, cuyos cursos de "Capstone Design" tuvieron duraciones de 1 y 2 semestres en ingeniería mecánica, ingeniería química e ingeniería de sistemas. El análisis con el VOSviewer identificó como palabra concurrente "experiencia” (Figura 5).

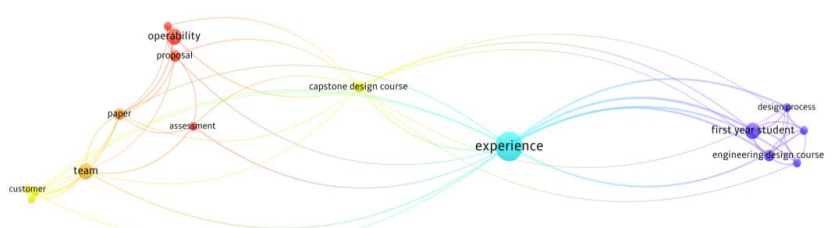

Figura 5. Análisis VOSviewer cuarto periodo. Fuente: Elaboración propia (2021)
Este periodo se caracterizó por investigar los efectos de la experiencia práctica del ejercicio profesional, lo que permitió mejorar la comprensión y relevancia dada a la documentación en los proyectos y procesos de diseño, e igualmente dar importancia a la generación de ideas para los proyectos (Bailey, 2007). En este periodo se resaltan como roles del profesor el de tutor y el de instructor. El de tutor como gestor y revisor del proyecto. El de instructor al proporcionar videos y herramientas para mejorar la experiencia del curso, el trabajo en equipos, los procesos de diseño y la interacción dentro del mismo (Powers y Summers, 2009).

Como factores que influían en desarrollo de los diseños se encontró, al igual de lo que se presenta a menudo en los proyectos de la industria, la claridad en la identificación de las necesidades del cliente; el saber hacer frente a las limitaciones económicas, de marketing, de seguridad y reglamentarias, al momento de realizar la propuesta de diseño (Al-Rizzo et al., 2010); la débil persistencia por parte de los jóvenes estudiantes; la falta de recursos didácticos; la colaboración y conformación de equipos; las visitas de campo, informes y exposiciones; las reuniones entre los tutores y los jóvenes estudiantes; aspectos fundamentales para mejorar la experiencia de diseño (Marlin, 2010).

En el quinto periodo comprendido entre 2011 y 2015, en la revisión bibliográfica se analizaron un total de 2 artículos, como se detalla en Tabla 8.

Tabla 8. Artículos periodo 2011-2015

\begin{tabular}{lc}
\hline Título & Cita \\
\hline $\begin{array}{l}\text { A conceptual framework to develop a project } \\
\text { management system with multidisciplinary } \\
\text { consilience in the capstone design course }\end{array}$ & (Kim, 2015) \\
$\begin{array}{l}\text { Transferability of practitioner-focused civil } \\
\text { engineering capstone design courses: An analysis } \\
\text { for a us and New Zealand approach }\end{array}$ & (Milke, Paul, y Koorey, 2015) \\
\hline & \multicolumn{2}{c}{ Fuente: Elaboración propia (2021) }
\end{tabular}

Los estudios de este periodo fueron realizados en Asia y Oceanía, donde los cursos de "Capstone Design" tuvieron una duración de 2 semestres en programas de ingeniería civil e ingeniería industrial. El análisis con VOSviewer identificó como palabras concurrentes "marco conceptual", "análisis" y "transferibilidad" (Figura 6). 


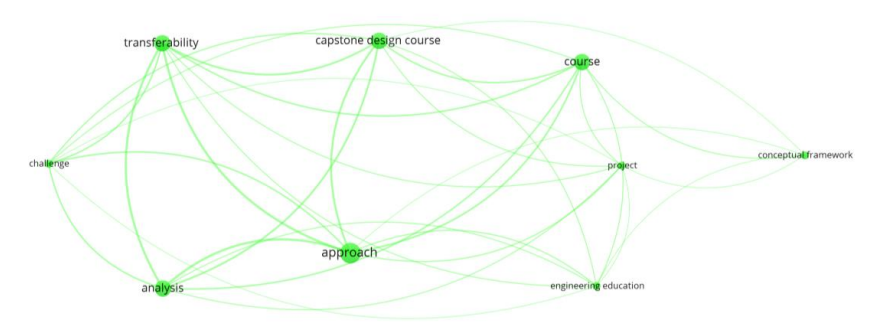

As vosviewe

Figura 6. Análisis VOSviewer quinto periodo. Fuente: Elaboración propia (2021)

Este periodo hace énfasis al marco conceptual de los "Capstone Desing", el cual debe ser multidisciplinar, además de promover el aprendizaje desde proyectos y competencias relacionadas con su gestión. Por su parte, como métodos de instrucción se incluyen el aprendizaje por indagación, el aprendizaje basado en problemas, el aprendizaje basado en proyectos, la enseñanza basada en casos, el aprendizaje por descubrimiento, entre otros. Los proyectos pueden ser formulados desde diferentes fuentes. Por los estudiantes a partir de necesidades del sector industrial; por los profesores a partir de sus proyectos de contratos privados o públicos; por la escuela de ingeniería; por exposiciones competitivas celebradas desde instituciones externas (Kim, 2015).

Los cursos de "Capstone Design", al igual que otros cursos de diseño y de ingeniería, deben proporcionar un equilibrio entre las habilidades "técnicas" y las habilidades "profesionales", al ser ambas deseadas por la industria. En ellos se brindan oportunidades de mejorar habilidades de comunicación entre grupos y jóvenes estudiantes, desde la elaboración de informes, cartas de presentación a empresas, presentaciones orales, informes semanales. Estos desafíos llevan a los jóvenes estudiantes a enfrentar su falta del pensamiento crítico y creativo que derivan en una mentalidad de diseño poco desarrollada. Es así que, se deben proponer proyectos abiertos, sin una sola respuesta "correcta"; plantear trabajos enfocados en los requisitos y recomendaciones del cliente, más que en cálculos detallados; y dibujar/bosquejar durante fases de diseño preliminar.
Como parte de las habilidades y desafíos desarrollados en los cursos, los jóvenes estudiantes obtuvieron información relevante para el proyecto, que no estaba disponible en los libros de texto guía o en las notas del curso, además de desarrollar su capacidad de tomar postura constructiva frente a su propio trabajo y el de sus compañeros de equipo u otros grupos (Milke, Paul, y Koorey, 2015). La preparación previa de los estudiantes antes del inicio del curso es muy importante para la conformación del equipo, la selección del proyecto, y la posterior aplicación de los resultados obtenidos en su futuro ejercicio profesional.

En el sexto periodo comprendido entre 2016 y 2020, en la revisión bibliográfica se analizaron un total de 6 artículos, como se detalla en Tabla 9.

Tabla 9. Artículos periodo 2016-2020

\begin{tabular}{ll}
\hline Título & Cita \\
\hline $\begin{array}{l}\text { Characterizing capstone design teaching: A functional } \\
\text { taxonomy }\end{array}$ & (Pembridge y Paretti, 2019) \\
$\begin{array}{l}\text { Project-based learning in capstone design courses for } \\
\text { engineering students: Factors affecting outcomes }\end{array}$ & (Joo, Lim, y Lee, 2019) \\
$\begin{array}{l}\text { A LabVIEW module to promote undergraduate research } \\
\text { in control of AC servo motors of robotics manipulator }\end{array}$ & (Yahya et al., 2020) \\
$\begin{array}{l}\text { Leveraging the capstone design experience to build self- } \\
\text { directed learning }\end{array}$ & (Paretti et al., 2020) \\
$\begin{array}{l}\text { Integrating digital civics into the engineering technology } \\
\text { classroom }\end{array}$ & (Bosman et al., 2020) \\
$\begin{array}{l}\text { Idea Generation Practices in a Biomedical Engineering } \\
\text { Capstone Course }\end{array}$ & (Ostrowski et al., 2020) \\
\hline
\end{tabular}
Fuente: Elaboración propia (2021)

Los artículos mencionados son trabajos realizados en la región de Asia y Norteamérica, con cursos de "Capstone Design" cuyas duraciones fueron de 1 y 2 semestres en ingeniería civil, ingeniería industrial, ingeniería aeronáutica e ingeniería química, entre otras. El análisis con VOSviewer identificó como palabras concurrentes "estudiante", "proyecto" y "problemas" (Figura 7

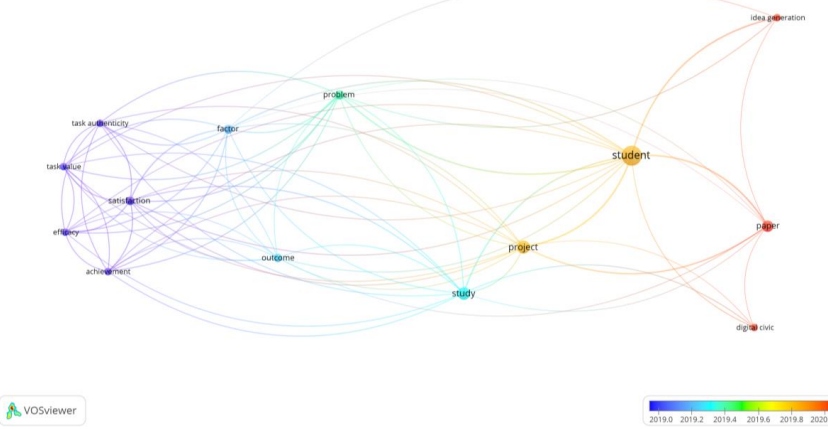

Figura 7. Análisis VOSviewer quinto periodo. Fuente: Elaboración propia (2021) 
Este periodo se caracterizó por la descripción exhaustiva de las prácticas pedagógicas utilizadas por el profesorado de diseño, desde una perspectiva funcional, lo que proporciona a los investigadores insumos para trabajos posteriores (Pembridge \& Paretti, 2019). Como en el cuarto periodo revisado, se proporcionan experiencias a los jóvenes estudiantes, aunque estas se centran en el aprendizaje (Yahya et al., 2020). Se resalta la necesidad de examinar los factores que afectan la satisfacción y el rendimiento en los cursos de diseño con los que se finaliza la formación en ingeniería (Joo, Lim, y Lee, 2019). Se replica un lugar de trabajo profesional de ingeniería para entrenar a los estudiantes en un ciclo completo que les ayuda a comprender el diseño como proceso social, en el que se hace hincapié en habilidades interpersonales, de trabajo en equipo, de comunicación técnica y de gestión de proyectos (Paretti et al., 2020). Se presenta la educación mediada por las Tecnologías de la Información y la Comunicación (TIC), como escenario para el desarrollo de un proyecto con la plataforma "Sprintbase"; en el que se dan recomendaciones para integrar eficazmente plataformas online a la enseñanza de la ingeniería (Bosman et al., 2020). Se muestra el diseño de prototipos y la generación de ideas como fundamental en los cursos de "Capstone Design" (Ostrowski et al., 2020).

Por otro lado, se reconoce que los instructores deben solucionar inconvenientes relacionados con la logística de la implementación del curso y con las estrategias de facilitación, para atender las dificultades que los estudiantes experimentan durante los procesos de resolución de problemas y de trabajo en equipo. Se requiere la identificación de los factores que afectan a la eficacia de los cursos para tenerlos en cuenta al momento de diseñarlos (Joo, Lim, y Lee, 2019). Se ha observado que los "Capstone Design" han promovido las asociaciones con la industria, el gobierno y el profesorado, para que los productos del curso reflejen la práctica de la ingeniería. Estos cursos promueven habilidades de comunicación a partir de la participación continua en la elaboración de informes, presentaciones técnicas, revisiones de diseño y demostraciones de prototipo (Paretti et al., 2020).

Finalmente, el diseño y creación de un prototipo de una base de datos con capacidad de búsqueda y de fácil uso y trabajo colaborativo en los Capstone, mejoran las habilidades de comunicación, persuasión, resolución de conflictos y solución de problemas (Bosman et al., 2020). Entre los retos que tienen los jóvenes estudiantes están la definición de problemas, la generación y evaluación de conceptos, el diseño detallado, generación de ideas; el atender en los procesos de fabricación y validación de los requisitos de diseño y comentarios del patrocinador (Ostrowski et al., 2020).

\section{Conclusiones}

La presente revisión de literatura permitió identificar el actual interés de publicación sobre los cursos de "Capstone Design" en revistas de ingeniería, especializadas en educación y especializadas en educación en ingeniería; interés que permite a la comunidad académica vinculada a los programas de ingeniería, contar con referencias para mejorar sus propuestas didácticas y curriculares. Se observa el compromiso que tienen los programas de ingeniería en asegurar que los estudiantes tengan una experiencia maestra de diseño, como experiencia culmen dentro de los currículos académicos, que integra principios, conceptos y técnicas vistas en cursos previos. Por ello, los cursos de experiencia mayor de diseño o "Capstone Design" deben proponer una estructura tal que permita cumplir con esta intencionalidad.

A finales de los años 80s se observó que la educación en la ingeniería no generaba profesionales aptos para la industria, por ende, era importante generar una solución, por lo que se crearon los cursos de diseño "Capstone Design". Estos cursos trajeron buenas experiencias a los futuros egresados, al fortalecer habilidades requeridas para desempeñarse en el sector industrial. En ellos, los estudiantes han de apropiarse de los proyectos, acompañados por instructores que facilitan, motivan y promueven 
la adquisición de habilidades importantes para un ingeniero como el aprendizaje autónomo o la comunicación escrita y oral.

Debido a las deficiencias que presentaban los recién egresados en habilidades blandas, como la poca comunicación asertiva, emergen los cursos de "Capstone Design" para mejorar estos aspectos. En este proceso, se vinculan las empresas, al brindar la oportunidad de hacer que los estudiantes participen en proyectos reales de ingeniería antes de su graduación. Los egresados dan gran importancia a estos cursos, dado que, en compañía de sus mentores, interactúan con ambientes en los cuales pueden asumir riesgo y de igual manera equivocarse para poder así simular situaciones reales de diseño.

Los cursos de "Capstone Design" han traído resultados positivos y gratificantes para los egresados, quienes participan en la planeación de proyectos y en el desarrollo de habilidades complementarias, necesarias para ejercer la ingeniería de manera exitosa. Así, estos cursos buscan mejorar las competencias blandas de los futuros profesionales y la calidad del diseño de los recién egresados, por lo que han evolucionado a través de los años para fortalecer en los jóvenes estudiantes habilidades personales e interpersonales, que potencien lo visto en la carrera y se formen excelentes profesionales, capaces de resolver problemas de la vida real.

\section{Agradecimiento}

Los autores agradecen al Semillero Modelado y Gestión de la Construcción de la Escuela de Ingeniería Civil del a Universidad Industrial de Santander, el cual es dirigido por el profesor Guillermo Mejía Aguilar, de donde recibimos todo el apoyo y capacitación para adelantar esta revisión de literatura. La investigación no fue financiada.

\section{Referencias Bibliográficas}

ABET, (2020). What is accreditation. Retrieved from https://www.abet.org/accreditation/ what-is-accreditation/
Abler, R. T., Owen, H. L., \& Riley, G. F. (2003). University methodology for internetworking principles and design projects. IEEE Transactions on Education, 46(2), 218-225. https://doi. org/10.1109/TE.2002.808239

Al-Rizzo, H., Mohan, S., Reed, M., Kinley, D., Hemphill, Z., Finley, C., Pope, A., Osborn, D., \& Crolley, W. (2010). Directional-based cellular e-commerce: Undergraduate systems engineering capstone design project. International Journal of Engineering Education, 26(5), 1285-1304. Andersen, D. A. (1992). Civil engineering capstone design course. Journal of Professional Issues in Engineering Education and Practice, 118(3), 279-283. https://doi.org/10.1061/(ASCE)10523928(1992)118:3(279)

Bailey, R. (2007). Effects of industrial experience and coursework during sophomore and junior years on student learning of engineering design. Journal of Mechanical Design, Transactions of the ASME, 129(7), 662-667. https://doi. org/10.1115/1.2722323

Banios, E. W. (1992). An Engineering Practices Course. IEEE Transactions on Education, 35(4), 286-293. https://doi.org/10.1109/13.168701

Bass, R. M., \& Begovic, M. M. (1997). A senior design course bridging power systems and power electronics: Power quality impacts of electric vehicle charging. IEEE Power Engineering Review, 17(2), 34. https://doi.org/10.1109/59.630441

Bosman, L., Daut, E., Van Winkle, R., \& Ricco, G. (2020). Integrating digital civics into the engineering technology classroom. Journal of Engineering Technology, 37(2), 20-32.

Brown, C. M., Sheppard, E. M., Vetelino, J. F., \& Galin, M. P. (1989). University-Industry Technology Interchange Through a Unique Engineering Projects Course. IEEE Transactions on Education, 32(3), 343-348. https://doi. org/10.1109/13.34167

Ekwaro-Osire, S. (2003). «Pan-Mentoring» as an Effective Element of Capstone Design Courses. International Journal of Engineering Education, 19(5), 721-724.

Gregson, P. H., \& Little, T. A. (1999). Using Contests to Teach Design to EE Juniors. 42(3), 19972000. https://doi.org/10.1109/13.779906 
Hamblen, J. O., Owen, H. L., Yalamanchili, S., \& Dao, B. (1999). An undergraduate computer engineering rapid systems prototyping design laboratory. IEEE Transactions on Education, 42(1), 8-14.

https://doi.org/10.1109/13.746325

Hanna, A. S., \& Sullivan, K. T. (2005). Bridging the gap between academics and practice: A capstone design experience. Journal of Professional Issues in Engineering Education and Practice, 131(1), 59-62. https://doi.org/10.1061/ (ASCE)1052-3928(2005)131:1(59)

Harris, T. A., \& Jacobs, H. R. (1995). On Effective Methods to Teach Mechanical Design. Journal of Engineering Education, 84(4), 343-349. https:// doi.org/10.1002/j.2168-9830.1995.tb00189.x Joo, Y. J., Lim, K. Y., \& Lee, S. Y. (2019). Project-based learning in capstone design courses for engineering students: Factors affecting outcomes. Issues in Educational Research, 29(1), 123140.

Joseph, J. (1999). Coordinating diverse set of capstone design experiments. Proceedings Frontiers in Education Conference, 1, 10-14. https://doi.org/10.1109/FIE.1999.839215 Kim, M. S. (2015). A conceptual framework to develop a project management system with multidisciplinary consilience in the capstone design course. Global Journal of Engineering Education, 17(2), 53-60.

Liebman, J. C. (1989). Designing the design engineer. Journal of Professional Issues in Engineering Education and Practice, 115(3). https://doi. org/10.1061/(ASCE)1052-3928(1989)115:3(261) Marin, J. A., Armstrong, J. E., \& Kays, J. L. (1999). Elements of an optimal capstone design experience. Journal of Engineering Education, 88(1), 19-22. https://doi.org/10.1002/j.2168-9830.1999. tb00405.x

Marlin, T. E. (2010). Teaching «operability» in undergraduate chemical engineering design education. Computers and Chemical Engineering, 34(9), 1421-1431.

https://doi.org/10.1016/j.compchemeng.2010.02.003

McEwen, E. E. (1994). Integrated capstone design experience. Journal of Professional Issues in Engineering Education and Practice, 120(2). Retrieved from

https://doi.org/10.1061/(ASCE)10523928(1994)120:2(212)

Mehta, S. I., \& Gronhovd, S. M. (1996). ication Modules on g Education. 39(3), 304-308. https:// doi.org/10.1109/13.538751

Mertz, R. L. (1997). A capstone design course. IEEE Transactions on Education, 40(1), 41-45. https://doi.org/10.1109/13.554668

Milke, M. W., Paul, M. J., \& Koorey, G. F. (2015). Transferability of practitioner-focused civil engineering capstone design courses: An analysis for a us and New Zealand approach. Australasian Journal of Engineering Education, 20(1), 1-8. https://doi.org/10.7158/D13-008.2015.20.1

Ostrowski, A. K., Daly, S. R., Huang-Saad, A., \& Seifert, C. M. (2020). Idea Generation Practices in a Biomedical Engineering Capstone Course. IEEE Transactions on Education, 63(2), 118-125. https://doi.org/10.1109/TE.2019.2915059

Paretti, M., Kotys-Schwartz, D., Ford, J., Howe, S., \& Robin, O. T. T. (2020). Leveraging the capstone design experience to build self-directed learning. International Journal of Engineering Education, 36(2), 675-686.

Pembridge, J. J., \& Paretti, M. C. (2019). Characterizing capstone design teaching: A functional taxonomy. Journal of Engineering Education, 108(2), 197-219.

https://doi.org/10.1002/jee.20259

Powers, L. M., \& Summers, J. D. (2009). Integrating graduate design coaches in undergraduate design project teams. International Journal of Mechanical Engineering Education, 37(1), 3-20. https://doi.org/10.7227/IJMEE.37.1.2

Richard, W. D., Taylor, D. E., \& Zar, D. M. (1999). A capstone computer engineering design course. IEEE Transactions on Education, 42(4), 288-294. https://doi.org/10.1109/13.804534

Russ Pimmel. (2001). Cooperative Learning Instructional Activities in a Capstone Design Course. Engineering Education, July.

https://doi.org/10.1002/j.2168-9830.2001. tb00621.x

The Joanna Briggs Institute. (2015). The Joanna Briggs Institute Reviewers'. Manual 2015: Me- 
thodology for JBI scoping reviews. Joanne Briggs Institute, 1-24. Recuperado a partir de http:// joannabriggs.org/assets/docs/sumari/ReviewersManual_Mixed-Methods-Review-Methods2014-ch1.pdf

Todd, R. H., \& Magleby, S. P. (2005). Elements of a successful capstone course considering the needs of stakeholders. European Journal of Engineering Education, 30(2), 203-214. https://doi. org/10.1080/03043790500087332

Todd, R. H., Sorensen, C. D., \& Magleby, S. P. (1993). Designing a Senior Capstone Course to Satisfy Industrial Customers. Journal of Engineering Education, 82(2), 92-100. https://doi. org/10.1002/j.2168-9830.1993.tb00082.x

Yahya, S., Moghavvemi, M., Almurib, H. A. F., \& Al-Rizzo, H. (2020). A LabVIEW module to promote undergraduate research in control of AC servo motors of robotics manipulator. Computer Applications in Engineering Education, 28(1), 139-153.

https://doi.org/10.1002/cae.22180 